\title{
NECESSIDADES BÁSICAS DAS ESPOSAS DE PACIENTES INFARTADOS NA FASE AGUDA DO TRATAMENTO
}

\author{
Edna Ikumi Umebayashi Takahashi * \\ Carmem de Almeida da Silva ** \\ Grazia Maria Guerra **
}

\begin{abstract}
RESUMO - Trata-se de um estudo exploratório que teve por finalidade as necessidades básicas das esposas de pacientes infartados, na fase aguda do tratamento. Foi utlizado como referencial para análise os conceitos de Nacessidades Básicas de MASLOW. Os resultados permitiram identificar como principais necessidades afetadas das esposas: segurança, amor e gregária, estima.
\end{abstract}

\begin{abstract}
The purpose of this study was to identify the basic need of the spouses of patients with myocardial infarction. The concepts of basic needs from MASLOW were used as conceptual framework. The data's abalysis showed the follwing nedds' affections of this population: safety, belongingness and love, esteem.
\end{abstract}

\section{INTRODUÇÃO}

As doenças cardiovasculares têm sido em nosso meio uma das principais causas de mortalidade, ocupando o Infarto Agudo do Miocárdio (IAM) um papel cada vez mais significativo dentro desse quadro LAURENTI ${ }^{4}$, LOLIO \& LAURENTI ${ }^{5}$. Para MELTEZER et alii e WIPPLE ${ }^{10}$ a doença coronariana acomete as pessoas no apogeu de sua vida produtiva, onde os indivíduos estão em pleno desenvolvimento de atividades profissionais e sociais.

Tais fatos associados parecem propiciar em nosso meio, um estigma em relação ao IAM, como uma condição limitante ou doença incapacitante.

Em seu estudo com coronariopatas, GUERRA \& $\mathrm{SILVA}^{3}$, constataram que a maioria da população é casada, o que implica que os indivíduos possuem família e essa constitue o elo entre o paciente e a sociedade. Para CHAVEZ \& FABER ${ }^{1}$ a famnia é a unidade social mais importante ao indivíduo, e como tal constitui o parâmetro dentro do qual a doença ocorre e se resolve. Os mesmos autores consideram que um membro doente ganha força de outros membros da familia. Entretanto, a interação família-paciente é frequentemente baseada em percepções situacionais que ambos não dividem, criando-se uma barreira e fazendo da visita uma fonte de stress.

Analisando aspectos da visita de familiares a coronariopatas, GUERRA, SILVA ${ }^{3}$ encontraram que a maioria dos visitantes são elementos da própria familia, ressaltando filhos $(39,2 \%)$ e esposas ou cônjuges $(31,3 \%)$ como os familiares que mais estiveram presentes no horário de visitas. TAKAHASHI ${ }^{9}$ por sua vez, verificou que $25,7 \%$ dos pacientes receberam visitas de cônjuges e 17,2\% receberam visitas de cônjuges acompanhados de outro familiar, o que caracterizou a presença do cônjuge como o visitante mais prevalente ao indivíduo acometido de IAM.

Para NYAMATHI ${ }^{8}$ a habilidade das esposas em lidar com seus comnpanheiros doentes tem um impacto significante não somente na saúde e funcionamer- nto da família como também na adaptação física e emocional à doença cardíaca.

Acreditamos que esse importante papel a ser desempenhado pela esposa pode, no entanto, ser ameaçado pela própria doença e suas repercussões no seio familiar.

Assim, consideramos como NYAMATHI ${ }^{8}$ que o diagnóstico do IAM é percebido como uma ameaça ao bem estar físico, pessoal e psicológico também pelos familiares. Para GAGLIONE ${ }^{2}$, em geral, a resposta da famnlia à doença caracterizada por choque que pode ser agravada pela sensação de morte. A mesma autora considera que a enfermeira tem o seu tempo e energia voltadas para as modalidades terapêuticas, dando menor atenção à famnlia. Desta forma, considera que a assistência aos membros da família requerem especial etenção assim como terapêutica pois constituem o suporte do sistema de apoio ao paciente.

A identificação das necessidades básicas dos familiares é assim, um importante aspecto da assistência de enfermagem. Com base neste pressuposto, estabelecemos para o presente estudo os seguintes objetivos:

- Identicar a percepção das esposas de pacientes com IAM, quanto a doença, internação e tratamento do seu familiar;

- Identificar as expectativas das esposas de doentes infartados a atuação do enfermeiro nessa área;

- Relacionar as necessidades básicas afetadas das esposas de pacientes com IAM, na fase do tratamento.

\section{METODOLOGIA}

I - População: Constou de 30 esposas de pa-

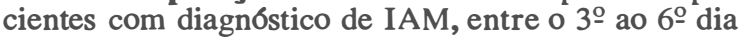
de internação em Unidade Coronariana de Hospital especializado em Cardiologia, no período de 11/04 a 12/06/89.

II - Coleta de Dados: a) Processo: Todas as es-

\footnotetext{
* Professor Assistente do Departamento de Enfermagem Médico-Cirírgica da Escola de Enfermagem da USP

** Enfermeiras da U.Co. do Instituto do Coração do Hospital das Clínicas da Faculdade de Medicina da USP
} 
posas de pacientes com IAM foram contactadas, esclarecidas a respeito do estudo e solicitadas a participarem do mesmo. Apos aceitação, aplicou-se o formulário em anexo (Anexo 1), tendo a entrevista ocorrido imediatamente após o horário de visita estabelecido. tos.

A duração média das entrevistas foi de 18 minu-

b) Instrumento utilizado: $O$ formulário constou de 3 partes específicas, assim, subdivididas:

Parte I - Identificação: Teve por finalidade a obtenção de algumas informações para caracterização tanto da esposa quanto do paciente. Em relação a esposa os seguintes dados de identificação foram incluídos: nome, sexo, idade, $\mathrm{n}^{\circ}$ de filhos, maioridade e grau de instrução. Quanto ao paciente, foram incluídos: $\mathrm{n}^{-}$ de internações anteriores em Unidades Coronarianas, dia de internação na UCo e condições clínicas monentâneas. Neste último item foram registrados dados referentes a presenÇa de artefatos terapêuticos como catéteres, drenos, venбclises, respiradores, monitores e outros, o item "Observaçōes" permitiu a anoração de outras situações familiares.

A Parte II do instrumento objetivou coletar dados sobre a percepção da esposa quanto a situação de doença, internação e tratamento familiar com IAM, permitindo assim a identificação de problemas, e a classificação dos mesmos segundo a concepção de $\mathrm{Ne}$ cessidades Básicas de MASLOW.

A Parte III teve opor finalidade identificar as espectativas das esposas quanto a atuação do enfermeiro na situação aguda de tratamento do paciente com IAM.

Consideramos que as expectativas quanto a atuação do enfermeiro explicitam as necessidades requeridas pelas esposas, tendo sido classificadas dentro do mesmo referencial das necessidades básicas já mencionado.

\section{III - Classificação dos problemas segundo} as NECESSIDADES BÁSICAS DE MASLOW* No presente estudo empregou-se como referencial teórico para a classificação dos problemas, a compreensão das necessidades básicas segundo Maslow. Este autor caracterizou as necessidades básicas como um dos aspectos da sua teoria da motivação humana, considerando ainda, a gratificação (satisfação e a frustação (insatisfação) da necessidade como elementos importantes dessa teoria, MOSCOVICI ${ }^{7}$.

Maslow concebe as necessidades básicas organizadas em categorias havendo uma hierarquia de predomínio entre elas, exemplificadora na figura abaixo:

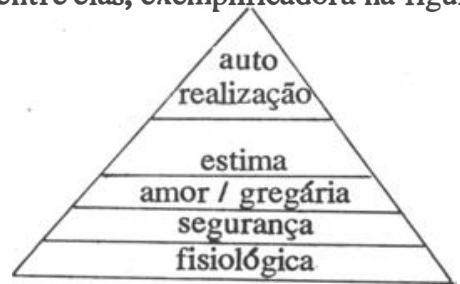

Considera entretanto que essa hierarquia não $\varepsilon$ rígida podendo variar sua posição e preponderência em função do grau de satisfação de uma determinada necessidade.

Para Maslow, a satisfação relativa de uma necessidade permitiria o surgimento de outras de nível mais elevado. Com a finalidade de facilitar a correlação e a classificação dos problemas, resumimos a seguir os preceitos fundamentais das diferentes necessidades básicas estabelecidas por Maslow.

Necessidades Fisiológicas: Relaciona-se aos processos corporreos de manutenção da homeostase ou seja, do esforço do organismo para manter um estado normal e constante. E a mais dominante de todas as necessidades sendo que uma condição de insatisfação de todas as necessidades, a fisiológica predominaria fazendo simplesmente inesistir as demais.

Necessidade de Segurança: Emerge como um novo quadro após a satisfação relativa da necessidade fisiologica. Entre alguns indicadores da necessidade de segurança do adulto normal podem ser citados: preferência por um trabalho estável; preferência por coisas familiares; seguro de vida, saúde; desejo de poupança e reservas para o futuro; procura de religião ou filosofia de vida - MOSCOVICI ${ }^{7}$. Ainda podem ser mencionados como outros indicadores da necessidade de segurança, a situação de dependência, de proteção, de medo, ansiedades e caos e assim por diante.

Necessidade Gregária e de Amor: Quando as necessidades fisiológicas e de seguirança estấo razoavelmente satisfeitas aparecem a necessidade gregária e de amor. Na presença desta, a pessoa sentirá, como nunca antes, a ausêncvia de amigos, namorados, esposas ou crianças; ela almejará por relações afetivas com pessoas em geral, por um lugar no grupo ou famnlia.

Necessidade de Estima: Todas as pessoas (excetuando alguns patologicos) tem uma necessidade ou desejo de avaliação firme e estável de si, de auto-respeito, auto-estima e estima dos outros. Os indicadores podem ser agrupados em duas categorias:

1) desejo de força, realização, suficiência, dominío e competência, confiança diante do modo, independência e liberdade.

2) dese jo de reputação e prestígio (respeito ou estima de outro), dominação, status, fama e glória, reconhecimento, atenção, importância, dignidade e apreço dos demais. A satisfação da necessidade de auto-estima conduz para os sentimentos de auto-confiança, valor, força, capacidade de ser útil e necessário para os outros. Sua frustação produz sentimento de inferioridade, fraqueza, abandono e desamparo. Ainda, a auto-estima mais saudável é baseada no merecido respeito de outros do que na fama e celebridade.

Necessidade de auto-realização: Quando todas as necessidades anteriores são satisfeitas surge uma necessidade mais elevada que é a tendência de efetivar suas potencialidades, como o sentido de atingir a plenitude do ser. maslow considera qinda, as necessidade básicas como motivos de deficiência sendo que, as necessidade de deficiência constituem no indivíduo, que precisam ser supridas de fora e por outros indivíduos para conservar a saúde, evitar de çurar doenças - MOSCOVICI ${ }^{7}$.

Assim sendo, a nível de assistência à saúde mais especificamente, de enfermagem, as necessidade identificadas a partir dos problemas apresentados pela clientela, constituiram aquelas que necessitariam de uma interveção de enfermagem.

* MASLOW, A. Motivation and Personality. 2 ed. New York: Harper\& Row, 1970. 


\section{RESULTADOS}

Os dados coletados através do formulário permitiram a obtenção e a apresentação dos seguintes resultados:

I - Caracterização da população

A - Esposas

B - Esposas x Paciente

II - Percepção da situação

III - Expectativas quanto a atuação do enfermeiro

IV - Necessidades bisicas afetadas

\section{I - CARACTERIzAÇÃo da populaçĀo}

A caracterização da população foi realizada com base nos dados biossociais das esposas (idade, grau de instrução, número de filhos) como também nas informaçōes quanto a situação de internação dos maridos (dia e número de internações, condiçōes clínicas momentâneas). Esses resultados são apresentados a seguir nas tabelas 1 a 4 .

\section{A. Esposas}

Tabela 1. Distribuição das esposas de pacientes infartados segundo idade e grau de instrução. São Paulo. 1989

\begin{tabular}{|c|c|c|c|c|c|c|c|c|c|c|}
\hline Idade & \multicolumn{2}{|c|}{$25 \longmapsto-35$} & \multicolumn{2}{|c|}{$35 \curvearrowleft 45$} & \multicolumn{2}{|c|}{$45 \longmapsto 60$} & \multicolumn{2}{|c|}{$60 \longleftarrow$} & \multicolumn{2}{|c|}{ TOTAL } \\
\hline instrução & № & $\%$ & № & $\%$ & № & $\%$ & № & $\%$ & № & $\%$ \\
\hline Analfabeta & & & & & 2 & 6,7 & 1 & 3,3 & 3 & 10,0 \\
\hline Saber ler e escrever & & & & & 1 & 3,3 & 1 & 3,4 & 2 & 6,7 \\
\hline Primário incompleto & & & & & 4 & 13,4 & & & 4 & 13,4 \\
\hline Primário completo & 1 & 3,3 & 1 & 3,3 & 3 & 10 & & & 5 & 16,6 \\
\hline 1\% grau completo & & & 2 & 6,7 & 2 & 6,7 & & & 4 & 13,4 \\
\hline 2 grau completo & & & 3 & 10 & 2 & 6,6 & & & 5 & 16,5 \\
\hline Universitário incompl. & 1 & 3,4 & 1 & 3,3 & & & & & 2 & 6,7 \\
\hline Universitário & 1 & 3,3 & & & 3 & 10 & 1 & 3,3 & 5 & 16,6 \\
\hline TOTAL & 3 & 10,0 & 7 & 23,3 & 17 & 56,7 & 3 & 10 & 30 & 100,0 \\
\hline
\end{tabular}

Pela tabela 1 verifica-se que a maioria das esposas encontra-se na faixa etária de 45 a 60 anos $(56,7 \%)$, sendo quem quanto ao grau de instrução, a população distribuiu-se de forma equivalente entre o primário completo, $2^{2}$ grau completo e universitário $(16,6 \%)$. Constata-se, porém, que mais da metade

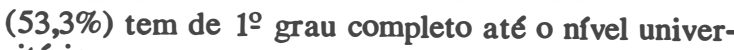
sitário.

Em relação a filhos, três das 30 esposas não têm filhos. Das 27 esposas com filhos, tivemos a seguinte distribuição quanto ao número e a maioridade dos mesmos:

Tabela 2. Distribuição das esposas de pacientes infartados segundo números de filhos e maioridade dos mesmos. São Paulo. 1989.

\begin{tabular}{|c|c|c|c|c|c|c|c|c|}
\hline № de Filhos & \multicolumn{2}{|c|}{$1 \longmapsto 2$} & \multirow{2}{*}{\multicolumn{2}{|c|}{$2 \longmapsto 4$}} & \multicolumn{2}{|c|}{ mais de 4} & \multicolumn{2}{|c|}{ T O T A L } \\
\hline Maioridade & № & $\%$ & & & $\mathrm{~N}$ & $\%$ & № & $\%$ \\
\hline menores de 18 anos & 7 & 25,9 & 3 & 11,1 & 1 & 3,7 & 11 & 40,7 \\
\hline maiores de 18 anos & 5 & 18,5 & 1 & 3,8 & 1 & 3,7 & 7 & 25,9 \\
\hline s. & 3 & 11,1 & 4 & 16,5 & 1 & 3,7 & 9 & 33,4 \\
\hline TOTAL & 15 & 56,5 & 8 & 33,4 & 3 & 11,1 & 27 & 100,0 \\
\hline
\end{tabular}

Constata-se nesta tabela que cerca de $55,5 \%$ das esposas possuem ate 2 filhos e destas $25,9 \%$ têm filhos menores de 18 anos e $18,5 \%$ têm filhos maiores de 18 anos. Do total de esposas com filhos, tamberm foi pre- valente a presença de filhos menores $(40,7 \%)$, o que pode ser mais um fator com implicação sócio-econômica à famnia acometida por problemas de saúde.

B. Esposas x Pacientes

Tabela 3. Distribuição da população segundo o dia de internação e o número de internação do paciente infartado em UCo. - São Paulo, 1989.

\begin{tabular}{|c|c|c|c|c|c|c|c|c|c|c|}
\hline \multirow{2}{*}{$\begin{array}{l}\text { no de } \begin{array}{c}\text { Dia de } \\
\text { internação } \\
\text { ninternaçöos }\end{array} \\
\end{array}$} & \multicolumn{2}{|c|}{30} & \multicolumn{2}{|c|}{$4^{\circ}$} & \multicolumn{2}{|c|}{ 5o } & \multicolumn{2}{|c|}{69} & \multicolumn{2}{|c|}{ TOTAL } \\
\hline & № & $\%$ & № & $\%$ & № & $\%$ & № & $\%$ & № & $\%$ \\
\hline $1^{\text {in }}$ internação & 10 & 33,4 & 11 & 36,6 & 5 & 16,9 & 2 & 6,5 & 28 & 93,3 \\
\hline $2^{\text {a } \text { internacão }}$ & - & & -3 & 3,3 & - & & -1 & 3,4 & 2 & 6,7 \\
\hline TOTAL & 10 & 33,4 & 12 & 39,9 & 5 & 16,9 & 3 & 9,8 & 30 & 100,0 \\
\hline
\end{tabular}

A tabela 3 mostra que para a maioria dos pacientes $(93,3 \%)$, esta era a primeira internação em Unidade Coronariana. Tal fato pode apresentar à familia uma situação diff́cil de hospitalização que tem como agravante, a mudança súbita no esquema de vida e a espeificidade de uma unidade altamente especializada.
O dia de internação dos pacientes foi determinado por critério anterior, onde considerou-se que apos o $3^{a}$ dia, os pacientes e familiares já teriam um reconhecimento inicial da situação de doença e internação. Por outro lado, foi considerado que os pacientes com evolução normal do quadro permanecem na unidade até 0 
5o ou 6 dia de internação. Com essa magrem de 4 dias, tornou-se possível incluir na população as esposas que não puderam comparecer com assiduidade às visitas.
$\mathrm{Na}$ população do presente estudo, prevaleceram as esposas cujos mardos encontravam-se no $3^{\circ}$ ou $4^{\circ}$ dias de internação $(33,4 \% 336,6 \%$, respectivamente).

Tabela 4. Distribuição da população segundo as condições clínicas momentâneas do paciente infartado. São Paulo, 1989.

\begin{tabular}{c|c|c}
\hline Condições & № & $\%$ \\
Clínicas & 15 & 50,0 \\
I & 10 & 33,3 \\
II & 05 & 16,7 \\
\hline III & 30 & 100,0 \\
\hline
\end{tabular}

\footnotetext{
Legenda: I - Monitorizado

II - Presença de até 2 artefatos terapêuticos

III - Presença de mais de artefatos terapéuticos
}

Quanto à avaliação das condições clínicas dos pacientes, relacionadas à presença de artefatos terapêuticos, verificou-sde que $50 \%$ das esposas tinham seus maridos apenas monitorizados, $33,3 \%$ com ate 2 artefatos terapéuticos e $16,7 \%$ com mais de 2 artefatos terapêuticos. Considerando que a classificação II correspondeu à presença de monitorização e venóclise, pode-se afirmar que a maioria dos pacientes encontra- va-se naquela momento, sob evolução natural do IAM.

\section{II - PERCEPÇĀO DA SITUAÇĀO}

Neste item procurou-se identificar o conhecimento da doença pela população e os problemas gerais e os prioritártios percebidos pelas esposas na fase aguda do tratamentop (tabelas 5, 6 e 7).

Tabela 5 - Distribuição da população segundo o que sabe em relação à doença que acometeu o marido. São Paulo, 1989.

\begin{tabular}{l|rc}
\hline \multicolumn{1}{c|}{ O QUE SABE EM RELAÇÃO } & & $\%$ \\
À DOENÇA & № & 76,7 \\
\hline É um infarto & 23 & 13,3 \\
É um infarto, e é grave & 4 & 10,0 \\
É um infarto, um entupimento da veia & 3 & 100,0 \\
\hline T O T A L & 30 & \\
\hline
\end{tabular}

Observa-se que todas as esposas que constituíram a população do estudo, souberam referir a doença, embora não se possa afirmar que todas soubessem exatamente do que se tratava o processo em si. Acredita-se que este fato talvez se deva em função de que as esposas foram participadas quanto ao diagnostico, e que seus maridos estavam internados em uma Unidade Coronariana, ou seja, unidade especializada no atendimento de pessoas com IAM. Além disso, outro fato que pode estar contribuindo para o conhecimento da doença, é o aumento da incidência de doenças cárdiocirculatórias nas últimas décadas, em especial nas grandes metrópoles, onde o processo de crescimento industrial e tecnologico evidenciam os agravantes dos fatores de risco. Parece existir uma relação entre a incidência da doença e a condição de vida em centros urbanos, favorecendo o surgimento de certo temos quanto a este diagnóstico. Assim, de alguma forma, as pessoas já ouviram falar do infarto e de sua evolução morbida, o que pode ser justificado pelos dados do presente estudo. A ausência de campanhas educativas oficiais populares permitem, entretanto, a divulgação de conceitos errôneos quanto a esta entidade nosologica. Observa-se tal fato presente na tabela acima, onde $10 \%$ da população tem um conceito errado do IAM. 
Tabela 6 - Problemas percebidos pelas esposas de pacientes infartados, na fase aguda do tratamento. São Paulo, 1989.

\begin{tabular}{l|rr}
\hline PROBLEMAS SENTIDOS & № & $\%$ \\
\hline - Estado subjetivo alterado (aflições, ansiedade, tristeza, aborrecimento, etc.) & 13 & 23,8 \\
- Medo (morte, outro infarto) & 10 & 18,8 \\
- Preocupação quanto ao futuro & 8 & 14,7 \\
- Não poder estar junto & 7 & 12,8 \\
- Acontecimento inesperado & 5 & 9,3 \\
- Pouco esclarecimento quanto ao tratamento e doença & 4 & 7,4 \\
- Responsabilidade assumidas por ausência do marido & 2 & 3,8 \\
- Não consegue dormir & 2 & 3,8 \\
- Dependência futura do marido & 1 & 1,4 \\
- Alteração na vida amorosa, afetiva, sexcual & 1 & 1,4 \\
- Condição económica difícil & 1 & 1,4 \\
- Buscando resposta na fé & 1 & 1,4 \\
\hline T O T A L & 55 & 100,0 \\
\hline
\end{tabular}

$\overline{\mathrm{x}}=1,9$ problemas/esposa

Os dados da tabela 5 mostram que os maiores percentuais recaem sobre as alterações do estado subjetivo $(23,8 \%)$, medo $(18,8 \%)$ e preocupação quanto ao futuro $(14,8 \%)$. Assim, observa-se a presença significativa de alterações emovionais nas esposas diante da situação de doença dos maridos. este fato vem confirmar a percepção da gravidade do quadro pelas esposas, associando-o às situações ameaçadoras e de um futuro incerto. NY AMATHI ${ }^{8}$ considera também que na presença do IAM, as esposas confrontam-se com um grande número de dificuldades, tais como a perda ou ameaça da perda do companheiro, trauma da separação da família e amigos, problemas domésticos e com os filhos, questões financeiras, mudanças no papel, futuro imprevisível, etc. Apesar da signiticativa referência das alterações do estado subjetico por esta população, verifica-se que as mencionadas por NYAMATHI ${ }^{8}$ estão, de certa forma, presentes.

Considerando o total dos problemas identificados nesse primeiro momento, pudemos obter uma média de 1,9 problemas mencionados por esposas.

Tabela 7. Problemas priorizados pelas esposas de pacientes infartados na fase aguda do tratamento. São Paulo, 1989.

\begin{tabular}{l|rr}
\hline PROBLEMAS PRIORITÁRIOS & \multicolumn{1}{c}{$\%$} \\
\hline - Medo (morte, reinfarto) & 13 & 13,4 \\
- Preocupação quanto à evolução e vida futura do marido & 8 & 20,5 \\
- Companheiro não estar bem & 5 & 12,8 \\
- Não poder estar junto & 4 & 10,3 \\
- Situação econômica difícil & 2 & 5,1 \\
- Insegurança quanto ao futuro da faminia & 2 & 5,1 \\
- Pouco esclarecimento quanto ao tratamento e doença do marido & 2 & 5,1 \\
- Responsabilidade perante a outra familia & 2 & 5,1 \\
- Ter que asusmir a direção dos negócios & 1 & 2,6 \\
\hline T O T A L & 39 & 100,0 \\
\hline$\overline{\mathrm{x}}=1,3$ problemas / esposa &
\end{tabular}

A tabela 7 revela que os problemas priorizados pelas esposas de pacierntes infartados na fase aguda, estão centrados no medo da morte e de outro infarto $(33,4 \%)$ e preocupação quanto à evolução e vida futura $(20,5 \%)$. Acredita-se que as atitudes em relação à morte estão embasadas em valores culturais, onde a perda e a morte não são aceitos e nem trabalhados, levando, muitas vezes, à omissão da própria equipe em lidar com este aspecto. Além disso, a qualidade de vida, sequelas e limitações advindas desta afecção, acarretam preocupação às esposas, uma vez que a nossa sociedade está centrada em valores de produção, sendo esta, a condição de aceitação ou marginalização do indivíduo.

\section{III - EXPECTATIVAS QUANTO À \\ ATUAÇĀO DO ENFERMEIRO}

Ao questionarmos as esposas sobre o que esperavam do enfermeiro, foram ressaltadas expectativas relacionadas tanto ao desempenho do profissional junto ao marido como também junto a si ou à família. A tabela 8 retrata as expectativas reveridas pela população. 
Tabela 8. Expectativas das esposas de pacientes infartados, quando à atuação do enfermeiro. São Paulo, 1989.

\begin{tabular}{lrrr}
\hline EXPECTATIVAS & No & $\begin{array}{r}\text { \% TOTAL \% TOTAL } \\
\text { GERAL }\end{array}$ \\
\hline PARCIAL
\end{tabular}

Em relação à tabela 8 , verifica-se que tanto as expectativas relacionadas ao marido quanto às relacionadas à própria esposa enfatizam a importância do papel expressivo do enfermeiro. Assim, atitudes humanísticas são as mais esperadas do enfermeiro. Do total de expectativas relacionadas a o amrido. o "cuidar bem" teve igual frequència $(40,7 \%)$ que as atitudes humanísticas. Em relação às pessoas, as atitudeds referentes ao papel expressivo representam $52,4 \%$ do total de suas expectativas, vindo a seguir a necessidade de "esclarecimento quanto às condições do marido" $(27,9 \%)$.

\section{NECESSIDADES BÁSICAS}

Tendo em vista a identificação dos aspectos que incomandam as esposas dos pacientes com IAM diante da doença, foi possível a identificação das necessidades básicas afetadas da população em estudo. Obteve-se, assim, o seguinte quadro:

- Estado subjetivo alterado*

- Medo (morte, reinfarto)*

- Preocupação quanto à evolução*

- Insegurança quanto ao futuro da famnlia

- Situação econômica difícil

- Pouco esclarecimento de fatos relacionados ao tratamento e doença

- Buscando resposta na fé

- Responsabilidade perante a outra famnlia

- responsabilidades assumidas por ausência do marido

- T er que assumir a direção nos negócios

- Dependência futura do marido

- Acontecimento inesperado

- Alteração na vida afetiva, amorosa, sexual

- Companheiro não estar bem

- Não poder estar junto

- Não poder estar junto

- Não consegue dormir NECESSIDADES BÁSICAS

* Problemas mais prevalentes (com frequência superior a 12,8\%) 
A análise dos problemas permitiu a correlação dos mesmos com as necessidades básicas estabelecidas por MASLOW. Observa-se aí que a necessidade de segurança, amor e gregária se relacionam com a maioria dos problemas, entretanto a necessidade de segu- rança abrange os problemas mais prevalentes.

Considerando as expectativas como aquilo que as esposas esperam do enfermeiro, optamos por correlacioná-las como necessidades a serem atendidas pelo enfermeiro, em busca do seu equilíbrio (Quadro 2).

Quadro 2. Necessidades básicas relacionadas às expectativas das esposas de opacientes infartados quanto à atuação do enfermeiro. São Paulo, 1989.

PROBLEMAS

Em relação ao marido $(*)$ :

- Papel expressivo: paciência, compreensão, atenção e humanidade $(* *)$

- Cuidar bem $(* *)$

- Não deixar que morra

- Acompanhar no que for necessário

- EDeixar ele andar

- Conversar com ele

Em Relação a sí

- Papel expressivo: compreensão, atenção, carinho, paciência, calor humano

apoio, amizade, amor, sinceridade, simpatia $(* *)$

- Esclarecimento quanto às condições do marido (**)

- Orientação quanto ao tratamento

- Transmita segurança

- Permissão para ficar mais tempo

NECESSIDADES BÁSICAS

Amor e Gregária

Segurança, Amor e Gregária, Estima

Estima.

Amor e Gregária.

Segurança

Amor e Gragária, Estima

(*) As necessidades aí relacionadas são pertinentes tanto ao marido quanto à esposa.

$(* *)$ Expectativas mais mencionadas

O quadro acima evidencia a presença significativa da necessidade de amor e gregária, estando ela relacionada à maioria das expectativas como também, abrangendo as expectativas mais mencionadas.

\section{CONCLUSÕES}

Os resultados apresentados permitem as seguintes conclusões:

- Todas as esposas souberam mencionar a causa de internação dos maridos, sendo que $76,7 \%$ referiu ser apenas um infarto, 13,3\% que é um infarto e é grave, e $10 \%$ que é um infarto, um entupimento de veia.

- Os problemas mais mencionados pelas esposas de pacientes infartadis, na fase aguda do tratamento, foram: alterações do estado subjetivo $(23,8 \%)$; medo $(18,8 \%)$; preocupação quanto ao futuro $(14,7 \%)$; não poder estar junto $(12,8 \%)$.

- Os problemas prioritários mais prevalentes foram: medo da morte e reinfarto $(33,4 \%)$, preocupação quanto à vida futura (20,5\%); companheiro não estar bem $(12,8 \%)$; não poder estar junto $(10,3 \%)$.

- As expectativas quanto à atuação do enfermeiro ressaltam opapel humanístico e expressivo com o mais esperado, tanto para atuação junto ao marido $(40,7 \%)$, quanto em relação à esposa $(52,4 \%)$. Tal papel inclui atitudes como: compreensão, atenção, carinho, ajuda, calor humano, paciência, amizade, amor.

- As necessidades básicas afetadas das esposas de pacientes infartados na fase aguda do tratamento foram: segurança, amor e gregária, estima e fisiologica.

- As necesidades afetadas relacionadas a partir das expectativas de atuação do enfermeiro foram: amor e gregária, segurança e estima.

\section{CONSIDERACÕES FINAIS}

As conclusões do presente estudo permitiram constatar mais uma vez, a necessidade de direcionar a atenção do enfermeiro a dois aspectos que têm sido relegados na prśatica hospitalar: a assistência à famnlia e o desempenho do papel expressivo pelo enfermeiro.

Entendemos que muitas vezes, a não incorporação de tais aspextos no âmbito da assistência de enfermagem advém das próprias condições em que essa prática é realizada e não necessariamente do desconhecimento da sua importância pelo profissional.

Acreditamos, entretanto, que a partir da devida valorização desses aspectos assistenciaids e da adequação das condições de realização da prática de enfermagem, será possível uma assistência sistemática e afetiva à famnlia, com o direcionamento da atenção do enfermeiro às áreas de desequilíbrio dos familiares como tamberm, ao atendimento das expectativas a ele atribuídas enquanto profissional de saúde.

Consideramos de vital importância que outros estudos venham a ser realizados, objetivando aprimorar cada vez mais o conhecimento dessa área carente de atuação pelo enfermeiro. 
Data:

Início;

Término:

\section{PARTE I: IDENTIFICAÇĀO}

\section{a) DO CONSORTE}

\section{NOME:}

SEXO:

IDADE:

№ DE FILHOS: menores de 18 anos:

maiores de 18 anos;

GRAU DE INSTRUÇĀO

\section{b) DO PACIENTE}

Dia de internação:

№ de internações em UC.:

Condições clínicas monentâneas - presença de dor, artefatos terapêticas ( Catéteres, drenos, venoclises, respiradores, monitores).

Observações: (outras implicações familiares)

\section{PARTE II: PERCEPÇĀO DA SITUAÇĀO}

A Sra. sabe que o seu marido teve (apresentou)? se sim, o quê?

Essaa situação ou condição do seu familiar está incomodando a Sra. de alguma forma? como e porquê?

O que mais a incomoda?

\section{PARTE III: EXPECTATIVAS QUANTO A ATUAÇĀO DO ENFERMEIRO}

O que a Sra. espera do enfermeiro nessa situação?

\section{REFERÊNCIAS BIBLIOGRÁFICAS}

1 CHAVEZ, FABER. Effect an education - orientation program on family members who visit their significant other in the intensive care unit. Heart Lung. Saint Louis, 16(1): 92-99, 1987.

2 GAGLIONE, M.K. Assessing and intervening with families of CCU patients. Bursing Clinics of North America. 19(3), Sept., P. 427, 1984.

3 GUERRA, G.M., SILVA, C.A. Caracterização da Visita na Unidade Coronariana, Papel do Enfermeiro. Trabalho apresentado no IX CONGRESSO PAULIS TA DE CARDIOLOGIA. Ribeirão Preto, 11 de junho de 1988. p. 9.

4 LAUREN TI, R. Epidemiologia das doenças cardiovasculares no Brasil. Arquivo Brasileiro Cardiologia. São Paulo, 38(4): 243-248, 1982.

5 LOLIO,' C.A.,' LAURENTI, R. Mortalidade por doença isquêmica do coreção no munićpio de São Paulo: evolução de 1950-81 e mudanças recentes na tendência.
Arquivo Brasileiro de Cardiologia. São Paulo, 46(3): 153-156, 1986.

6 MELTZER, L.E. et alii. Enfermagem na Unidade Coronariana. Rio de Janeiro: Atheneu, 1984, p. 5.

7 MOSCOVICI, F. desenvohimento interpessoal. 3 ed., Rio de Janeiro: Livros Técnicos e Científicos, 1985.

8 NYAMATHI, A.M. The coping responses of female spouses of patients with myocardial infarction. Heart Lung., Saint Louis, 16(1): P.86-92, 1987.

9 TAKAHASHI, E.I.U. Visitas de familiares a doentes infartados: análise de alguns parâmetros cardiovasculares. São Paulo, 1980. 69 p. (Dissertação de Mestrado - Escola de Enfermagem da USP).

10 WIPPLE, G.H. et alii. Insuficiência coronariana - assistência e tratamento. São Paulo: Editora Pedagógica e Universitária, 1980. p. 9. 